\title{
The impact of oligomeric anions on the speciation of protic ionic liquids
}

\author{
Mónia A.R. Martins a, Pedro J. Carvalho ${ }^{a}$, Luís M.N.B.F. Santos ${ }^{\mathrm{b}}$, Simão P. Pinhoc ${ }^{\text {, }}$ \\ João A.P. Coutinho ${ }^{\mathrm{a}, *}$ \\ ${ }^{a}$ CICECO - Aveiro Institute of Materials, Department of Chemistry, University of Aveiro, 3810-193 Aveiro, Portugal \\ ${ }^{\mathrm{b}}$ CIQUP, Department of Chemistry and Biochemistry, Faculty of Sciences, University of Porto, Porto, Portugal \\ c CIMO - Mountain Research Center, Polytechnic Institute of Bragança, 5300-253 Bragança, Portugal
}

\section{A R T I C L E I N F O}

\section{Article history:}

Received 11 October 2020

Revised 7 December 2020

Accepted 8 December 2020

Available online 11 December 2020

\section{Keywords:}

Protic Ionic Liquids

Carboxylates

Oligomeric anions

Acid:base

Ionicity

\begin{abstract}
A B S T R A C T
The synthesis of protic ionic liquids based in carboxylate anions, too often admitted as being straightforward, is actually challenging and must be carefully addressed. This review discusses the importance of oligomeric anions, in particular those based on carboxylates, in the behavior and nature of protic ionic liquids. There is strong evidence in the literature that the synthesis, and subsequent purification, of protic ionic liquids involving carboxylic acids, leads to structures in the liquid phase with an acid:base proportion different from the expected 1:1 stoichiometry. The formation of the oligomeric anions, mostly dependent on the proton transfer from the Brønsted acid to the Brønsted base, may lead to a higher ionicity that suggests the formation of true ionic liquids. It is here stressed the relevance of deepening the understanding of the interactions between the species and the speciation of the liquid phase, combining experimental and theoretical approaches to establish foundations for insightful advances in the area.
\end{abstract}

(c) 2020 Elsevier B.V. All rights reserved.

\section{Introduction}

By definition, protic ionic liquids (PILs) are an acid-base complex that results from a proton transfer from a Brønsted acid to a Brønsted base. The available proton on the cation allows for hydrogen bonding with the anion or other compounds [1-3]. It has been generally described to be a very simple [Acid][Base] structure, that is, a 1:1 stoichiometry, although it has been abundantly demonstrated that this often leads to low conductivities, i.e., bad PILs $[1,4]$.

When compared with aprotic ILs (APILs), PILs are economically advantageous due to their simple acid-base neutralization reaction, what makes them suitable for industrial large-scale use [5]. There are currently a number of examples at pilot or industrial scale of the use of these compounds. In the IonoSolv process protic ammonium-based ILs with the $\left[\mathrm{HSO}_{4}\right]^{-}$anion are used in detriment of APILs due to their technical and economic industrial benefits [6]. BASF, within their BASIL processes, uses PILs such as $[\mathrm{HMIm}] \mathrm{Cl}$ as an acid scavenger of $\mathrm{HCl}$ and [EMIm] $\left[\mathrm{HSO}_{4}\right]$ for liquid-liquid extraction [3], and at a pilot scale, Chrysalix Technologies uses a recyclable PIL as the solvent in the BioFlex process

\footnotetext{
* Corresponding author.

E-mail address: jcoutinho@ua.pt (J.A.P. Coutinho).
}

for the softwood and agricultural waste deconstruction [5]. A number of good reviews cover the properties and applications of PILs $[2,3,5]$.

Recently, while working on the synthesis and purification of a series of $\mathrm{N}, \mathrm{N}$-diethylethanolammonium carboxylates, we faced a somewhat unexpected situation [7]. The purification process by distillation under vacuum, since protic ILs can distill due to their high intrinsic volatility $[1,8,9]$, led to the formation of 'mixtures' with a 2:1 (acid:base) proportion as established by ${ }^{1} \mathrm{H}$ NMR spectra, instead of the planned 1:1 ratio. There are evidences in the literature $[1,8]$ showing the formation of an azeotrope at a concentration closer to the $2: 1$ stoichiometric proportion for some of these mixtures of strong basis with carboxylate acids, and Greaves and Drummond [2], on their review on PILs, stress the fact that the drying process under vacuum may remove more of one ion precursor than the other, leading to a nonstoichiometric mixture enriched in the less volatile component. But the fact that different mixtures $[1,7,8]$ seem to have azeotropes withing a narrow composition range around 2:1 raises questions and begs for a deeper understanding of interactions and speciation on the liquid phase responsible for this behavior.

The existence of acid salts of carboxylic acids, has been known for a long time [10]. Sodium hydrogen di-formate, $\mathrm{NaH}\left(\mathrm{HCO}_{2}\right)$, was first described by Bineau in 1847 [11], while sodium diacetate is a food additive widely commercialized as E262 [12]. Nevertheless, 


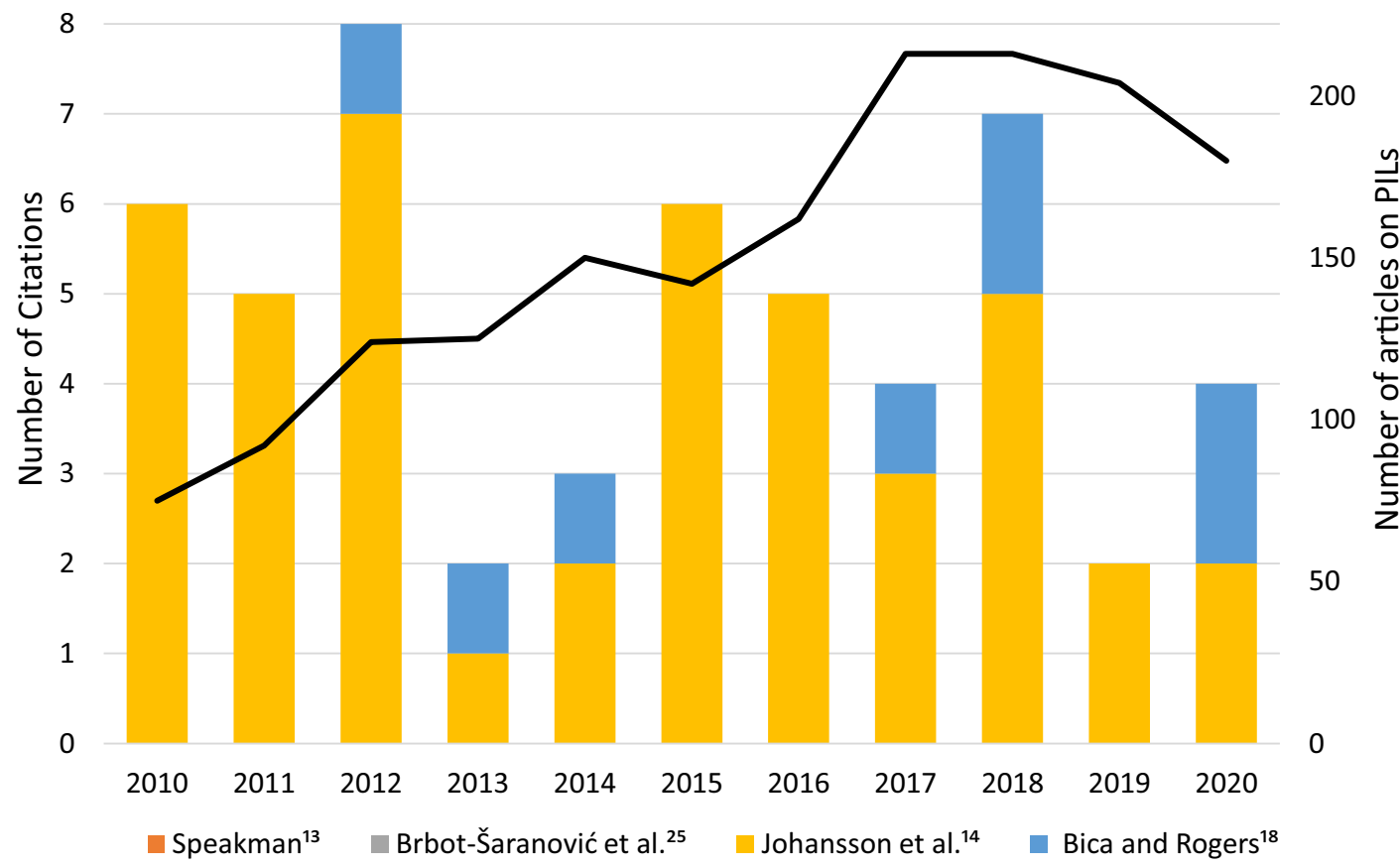

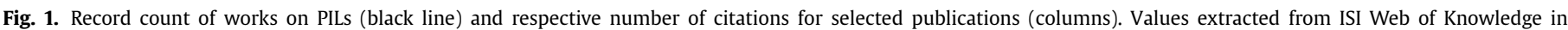
October, 2020.

as stated by Speakman "chemists often would not know of their existence, and few could have known of their commonness" [13]. Although most evidence related to these dimeric carboxylates (we will not use here the nomenclature suggested by Speakman [13] of dicarboxylates and dianions as it may lead to confusion) is based on their crystalline structures, and not their liquid phases, this suggests, nevertheless, that the 2:1 'mixtures' may not be simple mixtures of 1:1 PILs with excess acid but, instead, another form of PIL based on a different anion and thus, the structure of the ' $2: 1$ ' PILs in the liquid phase could probably be properly described as $\left[\mathrm{BH}^{+}\right]\left[(\mathrm{RCOO})_{2} \mathrm{H}^{-}\right]$.

Digging into the literature for evidences of the existence of dimeric carboxylates shows them to be far from unknown or even rare. Indeed, as will be argued below, many researchers have demonstrated or discussed the existence of dimeric carboxylate anions, clearly supporting the presence of these compounds even in the liquid phase. However, the comment by Speakman in his comprehensive and seminal 1972 review [13], stating the poor awareness of chemists on the existence of these structures, resonates true to someone familiar with the PIL literature. Despite some reports on this subject by McFarlane and co-workers $[4,14]$ and Rogers and co-workers [15-19], it seems that most of the community working with protic ionic liquids fails to recognize their existence, and to incorporate them on the analysis of their data as shown in the citation plot of Fig. 1.

This review aims to draw attention to the presence of oligomeric carboxylate anions in carboxylate-based PILs. In such PILs, very often the anionic structure in the liquid phase may actually be a dimeric carboxylate, and the resulting IL a $\left[\mathrm{BH}^{+}\right]\left[(\mathrm{RCOO})_{2} \mathrm{H}^{-}\right]$, or even higher oligomeric forms of the anion as suggested by McFarlane and co-workers [14], and further supported by the results of Johnson and co-workers [20], and not the expected $1: 1\left[\mathrm{BH}^{+}\right]\left[\mathrm{RCOO}^{-}\right]$salt. Although the dimeric carboxylate is rarely observed in aqueous salt solutions, since the presence of water favors the carboxylate formation to the detriment of the

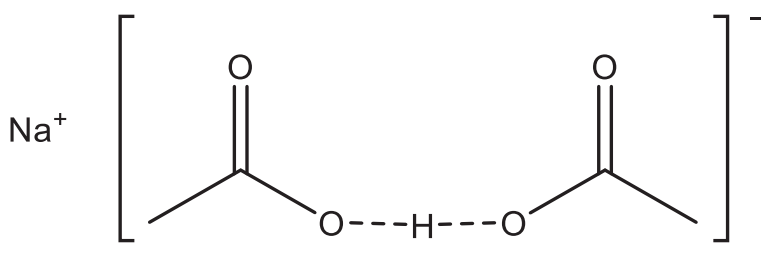

Fig. 2. Chemical structure of sodium hydrogen diacetate (CAS: 126-96-5).

oligomeric counterparts, the literature so far suggests that, in dry conditions, the dimeric carboxylate is often more stable than the carboxylate and thus ubiquitous on carboxylate based PILs [14].

\section{State of art}

As mentioned, acid salts of carboxylic acids have been known since mid-19th century, and quite a number of studies can be found in the literature during the early 20th century about the characterization of crystalline structures revealing the presence of $\left[\mathrm{BH}^{+}\right]\left[\mathrm{A}^{-}\right] \cdot[\mathrm{HA}]$, and in some cases also of $[\mathrm{B}] \cdot\left[\mathrm{BH}^{+}\right]\left[\mathrm{A}^{-}\right]$, in the solid phase [21-23]. The 1972 review of Speakman [13], although a milestone on this field, remains, regrettably, unfamiliar to many working with PILs. This work is remarkable for being not only a summary of the state of art on the subject at the time, but also because the author tries to systematize the knowledge on the field, proposing a classification for the various types of structures identified along the years, and also the nomenclature to be used to describe these compounds. That review covers a broader range of situations than those of relevance for this review, but clearly discusses the structure of the two carboxylates in the dimeric anion, described as crystallographically equivalent and linked by a short quasi-symmetric hydrogen bond involving the acidic hydrogen atom, giving origin to the complex anion [(RCOO)H(OOCR) $]^{-}$ [13], as exemplified in Fig. 2 for sodium diacetate. As for why the topic seems to be so poorly known in spite of its pervasiveness 
Speakman explains that acid salts of this type "were discovered, recorded in the literature, and often forgotten" [13]. In fact, by mid-20th century, more than one thousand structures had already been discovered, since most monocarboxylic acids yield acid salts, sometimes the acid salt crystallizing preferentially over the neutral salt [13].

Up to that moment works dealing with the dimeric anions were focused on their presence in the solid phase. The question about the structures of acid salts in the liquid phase was only addressed by the spectroscopic studies of Hadzi and co-workers [24,25]. The first of this works deals with hydrogen maleate, but it is their following article [25] that is more relevant in the context of this review. Here [25] the authors reported infrared and Raman spectra of tetramethylammonium or tetrabutylammonium acid salts of acetic acids. These salts would today be classified as aprotic ionic liquids. The spectroscopic study by Hadzi and co-workers [25] clearly demonstrates that the hydrogen bond in the dimeric carboxylate ions persists in the liquid state, and is of comparable strength to that in the corresponding crystals. However, its symmetry is, naturally, lost in the liquid phase. To which extent, and how does this affect the relative ionicities of the two carboxylate moieties, remains to be clarified.

Angell and co-workers were the first to use the designation "protic ionic liquids" [1] defined as a subgroup of ionic liquids formed by a proton transfer from a Brönsted acid to a Brönsted base [1]. This seminal article also contains the first reference to the possibility of forming dimeric anions on protic ionic liquids [1]. The authors show that $\alpha$-picoline with the strong protic acid trifluoromethanesulfonic forms an azeotrope close to 1:1 composition, while with trifluoroacetic acid the azeotrope occurs at around 2:1 (acid:base). This azeotrope is attributed to the formation of the dimeric anion $\left[(\mathrm{TFA})_{2} \mathrm{H}\right]^{-}$, that seems to have high stability on this system. The viscosities and conductivities at the 1:1 mixtures were measured and plotted in a Walden plot showing that systems formed by weak protic acids are 'weak' ILs [1]. But studies of conductivity show that this property increases strongly at acid rich compositions that favor the formation of the dimeric anion, becoming a 'good' IL. The field of PILs was thus off in a good foot with a detailed discussion of the stability of the dimeric anions as a result of a "strong hydrogen bond between the anion and the additional molecule of acid" [1] and how the maximum boiling points can identify them. Yet the result of this work seems to have known the fate of other acid salts works discussed by Speakman that "were discovered, recorded in the literature, and often forgotten" [13]. A typical example of this is the work by Treble et al. [26] that measured the conductivities of acetic acid-based PILs and observed a maximum in the conductivity-composition curves at high acetic acid mole ratios that they, without citing this previous work, hypothesized to result from the formation of conjugate anions $\mathrm{H}_{n} A_{n+1}$. Remarkably, much later, one of the authors of that study, K.E. Johnson [20] would demonstrate the origin of the maxima in conductivity of the PILs to be indeed related to the formation of "complexes" $\left[(\mathrm{HA})_{\mathrm{n}} \cdot \mathrm{A}\right]^{-}$, even if they do not provide an adequate characterization of the nature of this "complex". And while a number of works, mostly from McFarlane or Rogers Labs, cite the possibility of formation of dimeric and oligomeric anions [4,14-19], few works dealing with PILs contemplate this hypothesis in the analysis of the reported behaviors of the PILs studied.

Along with Angell [1], one of the most important set of works on PILs is by MacFarlane [14]. In 2008 he and his co-workers reported an essential study on PILs with "dimeric and oligomeric" ions [14]. Although based essentially on a fluidity and conductivity study of $\mathrm{N}$-methylpyrrolidine-acetic acid mixtures at different acid:base proportions, the authors show that the equimolar system presents a low degree of ionicity, or weaker degree of proton transfer, while the 2:1 (acetic acid: $N$-methylpyrrolidine) displays the highest viscosity, with the conductivity maximum observed at even higher acetic acid concentrations. They suggest that ionicity increase with concentration, in the $1: 1$ to $2: 1$ region, is related to the formation of dimers $\left[\mathrm{CH}_{3} \mathrm{COO} . \mathrm{CH}_{3} \mathrm{COOH}\right]^{-}$or even oligomeric $\left[\left(\mathrm{CH}_{3} \mathrm{COO}\right)_{x} \mathrm{H}_{x-1}\right]^{-}$anions stabilized by hydrogen bonds [14]. Quantum chemical calculations suggest that the oligomeric acids are more stable than the monomeric species, and that while both the monomer and the dimer of acetic acid form stable acid:base complexes with the $N$-methylpyrrolidine, the monomer, unlike the dimer, prefers to retain its neutral form in the complex. This remains the most comprehensive study of a PIL at a stoichiometry different from $1: 1$, and the most crucial evidence that carboxylatebased PILs will often form at stoichiometries other than 1:1. In spite of its relevance, this work remains only moderately cited as shown in Fig. 1, and seldom for the main results there reported. Also of major significance is the final discussion on the nature of the PILs formed with oligomeric ions: are they ionic liquids or saltsolvent mixtures? The lack of spectroscopic studies on the nature of the dimeric carboxylate formed precludes a formal answer but based on the evidence collected by Hadzi and co-workers [25] discussed above, it seems clear that the 2:1 PIL is more an ionic liquid than its 1:1 counterpart that will have a much larger amount of neutral species present. To this date, detailed spectroscopic studies of PILs at stoichiometries other than 1:1 seem to be lacking for a deeper understanding of these fluids. It is also striking that MacFarlane failed in subsequent works to explore these results further, and seldom in his work PILs of other stoichiometries are studied.

Rogers and co-workers were the only group of researchers working with PILs that would consistently use the dimer formation concept [15-19] in the interpretation of their data, often leading them to significant advances. The first work worth mentioning here is an attempt at liquefying active pharmaceutical salts through a strategy that Bica and Rogers called "confused ionic liquids" [18]. Their idea was to liquefy salts by mixing them with a solid acid, or base, to form oligomeric ions. The authors are very familiar with the previous literature on the subject (see refs. [8-12] of their article), and successfully demonstrate the concept by liquefying tetrabutylphosphonium salicylate and lidocainium salicylate. NMR spectra clearly indicate the formation of the dimeric tetrabutylphosphonium disalicylate. Gurau et al. [19] further showed the formation of diacetate-based ILs upon $\mathrm{CO}_{2}$ absorption by ethylimidazolium acetate.

Rogers and co-workers published in 2013 a very insightful perspective on the effect of the ionicity in salts solvates and ionic liquids, and how this was critical to the understanding of their behavior [15]. That perspective is very broad in scope, discussing some of the results already presented here and a few new cases. Unfortunately, it has a strong focus on the solid phase with the use of crystal structures to understand the molecular interactions present. A particularly relevant compound, not discussed elsewhere, is an aprotic ionic liquid, 1,2,3-trimethyimidazolium disalicylate, that seems to be an uncommon intermediate between the crystals of acid salts of type A and type B (according to the classification of Speakman [13]) in which both salicylates seem to have a degree of ionicity but are asymmetric, one of them clearly less ionic than the other, thus being, probably, a pseudo-type A acid salt [13].

Given the history of their research on PILs of uncommon stoichiometries, it is thus not surprising to see Rogers and co-workers [17] expressing their frustration at the thoughtless way in which Banerjee and co-workers [27-31] classified CAGE, choline geranate with a 2:1 stoichiometry, first as ionic liquid and then as deep eutectic solvent (DES) to match the hot topic of the day. The relevance of this question is much more than just a matter of correct terminology. As Rogers and Gurau [17] correctly point it is a failure to recognize the true nature of the compound under study that 
"prevents a mechanistic understanding of how this system works". The title of their 2013 review [15] is also clear about their feelings and vision on this subject. Unfortunately, Banerjee and co-workers were unable to understand their concerns and to address them adequately [27]. The results summarized on this perspective suggest that the 2:1 choline geranate may actually be formed by a dimeric anion of digeranate being a true ionic liquid in agreement with the suggestion of Rogers and Gurau [17] that call it an "IL with a complex anion". However, spectroscopic results to confirm this hypothesis are still required.

Another extensive study of ion oligomerization in protic ionic liquids was reported by Berton et al. [16]. The authors show how moving away from the fixed conventional 1:1 stoichiometry they can get an extra degree of freedom that would allow the tuning of the thermophysical properties of the protic ionic liquid and its solvating abilities. It is particularly relevant for the object of this review the study of the mixture of butylammonium acetate with acetic acid where the results are interpreted under the light of the formation of the diacetate anion, although no hard evidence for the presence of the dimeric anion is reported.

The tunability of the ILs, both protic and aprotic, by mixing with the precursors of their anions, proposed first by Bica and Rogers [18] and then by Berton et al. [16], allows an extra degree of freedom in the design of these compound. It is then possible to match their properties and performance to the requirements of the processes and products to be created or optimized. This is similar to the idea recommended by us for DES [32]. Instead of fixed stoichiometries, that at the end of the day have very little adhesion to any subjacent molecular reality, mixtures of hydrogen bond donors and hydrogen bond acceptors that match the requirements of an application should be used. It would be important to think of PILs and DES not as two different realities but as two extremes of a spectrum of proton sharing (from simple sharing on a hydrogen bond to full transfer in a PIL). And also to keep in mind when working with DES systems that, unlike aprotic ILs and true PILs, where the proton transfer is essentially complete, these are mixtures and not pure compounds as the use of a name, be it reline or pyridinium acetate, may deceptively induce the user to believe.

For a matter of simplicity and also due to the available data on the subject, this review has been emphasizing the dimeric anion, but other oligomeric anions are also possible, and in some cases, as shown by Johnson and co-workers [20], apparently without a clear stoichiometry. Cases of higher, well-defined stoichiometries have also been reported, a notorious case being the system acetic acid-triethylamine $[33,34]$. This was first reported by van Klooster and Douglas in 1945 [33]. It is a curious system since the two compounds are immiscible in the liquid phase, yet presents an unlikely azeotrope with a maximum in temperature that suggests the formation of a new compound(s?) in the system. It has been the target of a detailed study by Kohler et al. [34] that established the stoichiometry of the complex as $3: 1$. This system seems prone to rediscovery. Lv et al. [35] seemed completely oblivious to previous works on the subject when they report their study on the equilibrium of triethylammonium acetate by IR spectroscopy. Although this work did not bring new light on the subject it prompted Rogers and co-workers [36] to revisit this system with conclusions different from those by Kohler et al. [34] regarding the stoichiometry of the complex. In any case, all works concur into the notion that a complex oligomeric anion forms on this system. Another study by Kohler and co-workers [37] further testifies on how rich and complex these systems of carboxylic acids with amines can be, leaving space for them to be revisited today under the light of the protic ionic liquid studies, and with the novel and more powerful techniques available today, including molecular simulations [35].

The 2013 perspective [15], and all the work by Rogers and coworkers [15-19] on the subject of oligomeric anions, provide some evidences and answers, but bring up other questions. One of the most relevant is about the nature of the oligomeric anion. Although it is clear in some situations, in particular from their crystallographic structures reported Gurau et al. [19] and the NMR of Bica and Rogers [18] that the dimeric anion is a real entity, often the authors mention, in relation to other situations, the formation of "noncoordinating oligomeric anions" [15]. These would be completely different in nature from the dimeric anions whose presence is well established in the solid phase, were reported by Hadzi and co-workers [25], and are also patent in the NMR of Bica and Rogers [18] for the tretrabutylphosphonium disalicylate. In any case, a more careful and comprehensive study of the nature of these complex anions using vibrational spectroscopy or other adequate technique is required. The set of experimental analysis and techniques to apply is not yet clear. Definitely, those based on resonance or spectroscopy studies are very significant. However, properties such as density, viscosity, refractive index, the heat of mixing, acoustical attenuation, and permittivity measurements can give hints on the structure of the main species in the solution as tentatively reported in the works of Orzechowski et al. [38,39] by studying mixtures of propionic acid and triethylamine.

\section{Conclusions and perspectives}

The results discussed here suggest that the synthesis of protic ionic liquids based in carboxylate anions (and probably the synthesis of protic ionic liquids in general, as the dimeric anions are not restricted to dicarboxylate ions [18]) is more challenging than generally admitted, and should be addressed carefully. As stated by Rogers and coworkers [15], due to oligomerization the product of mixtures of Brönsted acids and bases "are not necessarily what would be predicted based on the stoichiometry of the reactants". In many situations, the structure present in the liquid phase is an acid salt, and thus, one must not think in the resulting IL as a 1:1 acid-base structure. As observed in many works, the possibility of obtaining a 2:1 or even a form with a higher-order stoichiometric ratio structure as the most stable combination is plausible, and often likely.

A conclusion to this review is that the ionic liquids community, particularly the authors working with PILs, has been too much focused on preparing and studying 1:1 mixtures, neglecting the fact that very often the dimeric anions may form more stable structures than the single anion. Thus the closer form to a true ionic liquid, with the maximum ionicity, maybe the 2:1 stoichiometry, or even, in some cases, higher-order stoichiometries. To convey this message was the main reason that prompted us to write this account. We can only hope that in the future more comprehensive works taking into account the possibility of stoichiometries other than 1:1 will become more common.

There are also quite some outcomes of the considerations pondered on this work that are worth exploring in the future. The consequence that one should not focus all the work on PILs on the $1: 1$ structures, is that more comprehensive studies covering other compositions are required. Although it would be easy or convenient to explore just a few alternative stoichiometries, we believe that the most relevant studies will come from scanning the entire concentration range, between the pure acid and pure base. This is what allowed Hadzi [25], Angell [1], Johnson [20], Rogers [16], and even us [40], to foster the understanding of these systems. It is not clear, at this point, what are the main properties that should be explored to understand which the dominant species present in the system are. The VLE, with the identification of the azeotropic point, suggested by Angell and co-workers [1], would certainly be useful. Still, it is experimentally very taxing, and the results by Ribeiro et al. [8] suggest that the differences in volatility between the two precursors may mask the composition of the dominant species. 
However, the knowledge of the VLE is important if evaporation or distillation is to be used to purify the PIL after the synthesis, and NMR or any other suitable analytical technique should always be used to ascertain the composition of the final mixture before using it. In any case, VLE studies should be coupled to those of other thermophysical properties, as suggested below, and as reported by Angell and co-workers [1], to establish the relationships between the various macroscopic properties and the liquid phase speciation.

An alternative that would probably be accessible to more researchers is the measurement of densities, viscosities, and conductivities in the entire concentration range. The viscosity seems to be a good guide to identify the dominant complexes present [4], in particular when coupled with the conductivity and Walden plots [4]. Johnson and co-workers [20], recently proposed another approach using essentially the same data, yet for lack of available information at that time, it has been applied only to a handful of systems. More extensive measurements of thermophysical properties, coupled with spectroscopic studies (both NMR as suggested by Bica and Rogers [18], or vibrational spectra as carried out by Hadzi and co-workers [25]), could lead to a much better understanding of PILs. In particular, clarify the role that dimeric and oligomeric anions play on their speciation, and establish a more precise image of the nature of these ions in the liquid phase, as their presence and form in the solid phase have been well-established since long (though often forgotten) [13]. Naturally, simulation techniques should be coupled to these experimental approaches to complement them and achieve a deeper understanding of the nature of species present. This is particularly relevant for spectroscopic studies on this field. The rich nature of these systems and the sort of hydrogen bond behind the formation of the oligomeric anions will certainly yield most rewarding results to those that may wish to engage in this quest.

\section{Declaration of Competing Interest}

The authors declare that they have no known competing financial interests or personal relationships that could have appeared to influence the work reported in this paper.

\section{CRediT authorship contribution statement}

Mónia A.R. Martins: Investigation, Resources, Writing - original draft, Writing - review \& editing, Visualization. Pedro J. Carvalho: Investigation, Writing - review \& editing. Luís M.N.B.F. Santos: Investigation, Writing - review \& editing. Simão P. Pinho: Investigation, Writing - review \& editing. João A.P. Coutinho: Conceptualization, Investigation, Writing - original draft, Writing - review \& editing, Supervision, Project administration, Funding acquisition.

\section{Acknowledgements}

This work was developed within the scope of the project CICECO-Aveiro Institute of Materials, UIDB/50011/2020 \& UIDP/50011/2020, CIMO-Mountain Research Center, UIDB/00690/2020, and CIQUP, University of Porto (Projects: PEstUID/QUI/00081/2013) financed by national funds through the Portuguese Foundation for Science and Technology (FCT)/MCTES. This work was also developed within the scope of the IndoPortuguese Program for Cooperation in Science \& Technology DST/INT/Portugal/P-01/2017, financed by FCT and the Government of India. P.J.C. acknowledge FCT for his contract under the Investigator FCT 2015 contract number IF/00758/2015.

\section{References}

[1] M. Yoshizawa, W. Xu, C.A. Angell, Ionic liquids by proton transfer: vapor pressure, conductivity, and the relevance of $\Delta \mathrm{pKa}$ from aqueous solutions, J. Am. Chem. Soc. 125 (2003) 15411-15419, doi:10.1021/ja035783d.
[2] T.L. Greaves, C.J. Drummond, Protic ionic liquids: evolving structure-property relationships and expanding applications, Chem. Rev. 115 (2015) 11379-11448, doi:10.1021/acs.chemrev.5b00158.

[3] T.L. Greaves, C.J. Drummond, Protic ionic liquids: Properties and applications, Chem. Rev. 108 (2008) 206-237, doi:10.1021/cr068040u.

[4] J. Stoimenovski, E.I. Izgorodina, D.R. MacFarlane, Ionicity and proton transfer in protic ionic liquids, Phys. Chem. Chem. Phys. 12 (2010) 10341-10347, doi:10 1039/c0cp00239a.

[5] M.B. Shiflett (Ed.), Commercial Applications of Ionic Liquids, Springer International Publishing, 2020, doi:10.1007/978-3-030-35245-5.

[6] A. George, A. Brandt, K. Tran, S.M.S.N.S. Zahari, D. Klein-Marcuschamer, N. Sun, N. Sathitsuksanoh, J. Shi, V. Stavila, R. Parthasarathi, S. Singh, B.M. Holmes, T. Welton, B.A. Simmons, J.P. Hallett, Design of low-cost ionic liquids for lignocellulosic biomass pretreatment, Green Chem. 17 (2015) 1728-1734, doi:10. 1039/c4gc01208a.

[7] M.A.R. Martins, G. Sharma, S.P. Pinho, R.L. Gardas, J.A.P. Coutinho, P.J. Carvalho, Selection and characterization of non-ideal ionic liquids mixtures to be used in $\mathrm{CO}_{2}$ capture, Fluid Phase Equilib. 518 (2020) 112621, doi:10.1016/j.fluid.2020. 112621.

[8] F.M.S. Ribeiro, C.F.R.A.C. Lima, A.M.S. Silva, L.M.N.B.F. Santos, Experimental evidence for azeotrope formation from protic ionic liquids, ChemPhysChem. 19 (2018) 2364-2369, doi:10.1002/cphc.201800335.

[9] F.M.S. Ribeiro, C.F.R.A.C. Lima, I.C.M. Vaz, A.S.M.C. Rodrigues, E. Sapei, A. Melo, A.M.S. Silva, L.M.N.B.F. Santos, Vaporization of protic ionic liquids derived from organic superbases and short carboxylic acids, Phys. Chem. Chem. Phys. 19 (2017) 16693-16701, doi:10.1039/c7cp02023f.

[10] J.C. Speakman, H.H. Mills, 230. The crystal structures of the acid salts of some monobasic acids. Part VI. Sodium hydrogen diacetate, J. Chem. Soc. (1961) 1164-1175, doi:10.1039/jr9610001164.

[11] M.A. Bineau, Note sur les biformiates de potasse et de soude, Ann. Chim. Phys. (1847) 291-296.

[12] H. Mohammadzadeh-Aghdash, Y. Sohrabi, A. Mohammadi, D. Shanehbandi, P. Dehghan, J. Ezzati Nazhad Dolatabadi, Safety assessment of sodium acetate, sodium diacetate and potassium sorbate food additives, Food Chem. 257 (2018) 211-215, doi:10.1016/j.foodchem.2018.03.020.

[13] J.C. Speakman, Acid salts of carboxylic acids, crystals with some "very short" hydrogen bonds, Struct. Bond. 12 (1972) 141-199, doi:10.1007/BFb0117543.

[14] K.M. Johansson, E.I. Izgorodina, M. Forsyth, D.R. MacFarlane, K.R. Seddon, Protic ionic liquids based on the dimeric and oligomeric anions: $\left[(\mathrm{AcO})_{\mathrm{x}} \mathrm{H}_{\mathrm{x}-1}\right]^{-}$, Phys. Chem. Chem. Phys. 10 (2008) 2972-2978, doi:10.1039/b801405a.

[15] S.P. Kelley, A. Narita, J.D. Holbrey, K.D. Green, W.M. Reichert, R.D. Rogers, Understanding the effects of ionicity in salts, solvates, co-crystals, ionic cocrystals, and ionic liquids, rather than nomenclature, is critical to understanding their behavior, Cryst. Growth Des. 13 (2013) 965-975, doi:10.1021/ cg4000439.

[16] P. Berton, S.P. Kelley, H. Wang, A.S. Myerson, R.D. Rogers, Separate mechanisms of ion oligomerization tune the physicochemical properties of nbutylammonium acetate: cation-base clusters vs. Anion-acid dimers, Phys. Chem. Chem. Phys. 19 (2017) 25544-25554, doi:10.1039/c7cp04078d.

[17] R.D. Rogers, G. Gurau, Is "choline and geranate" an ionic liquid or deep eutectic solvent system? Proc. Natl. Acad. Sci. U. S. A. 115 (2018) E10999, doi:10.1073/ pnas.1814976115.

[18] K. Bica, R.D. Rogers, Confused ionic liquid ions - a "liquification" and dosage strategy for pharmaceutically active salts, Chem. Commun. 46 (2010) 12151217, doi: $10.1039 / \mathrm{b} 925147 \mathrm{~b}$.

[19] G. Gurau, H. Rodríguez, S.P. Kelley, P. Janiczek, R.S. Kalb, R.D. Rogers, Demonstration of chemisorption of carbon dioxide in 1,3-dialkylimidazolium acetate ionic liquids, Angew. Chemie - Int. Ed. 50 (2011) 12024-12026, doi:10.1002/ anie.201105198.

[20] N.P. Aravindakshan, K.E. Gemmell, K.E. Johnson, A.L.L. East, The origin of the conductivity maximum vs. mixing ratio in pyridine/acetic acid and water/acetic acid, J. Chem. Phys. 149 (2018) 094505, doi:10.1063/1.5039623.

[21] E.A. O'Connor, XLIX.-The binary system, aniline-acetic acid, J. Chem. Soc. Trans. 119 (1921) 400-403, doi:10.1039/CT9211900400.

[22] E.A. O'Connor, CLXXIX.-Some binary system composed of acetic acid and the homologues of aniline, J. Chem. Soc., Trans. 125 (1924) 1422-1427, doi:10.1039/ CT9242501422.

[23] W.W. Lucasse, R.P. Koob, J.G. Miller, The solid-liquid phase equilibria of the system p-toluidine-acetic acid, J. Phys. Chem. 48 (1944) 85-89, doi:10.1021/ j150434a005.

[24] F. Avberlj, M. Hodošček, D. Hadži, Vibrational analysis of the hydrogen maleate ion using scaled ab initio force constants, Spectrochim. Acta Part A Mol. Spectrosc. 41 (1985) 89-97, doi:10.1016/0584-8539(85)80088-X.

[25] A. Brbot-Šaranović, D. Hadži, M. Hodošček, B. Orel, Vibrational spectra of some tetraalkylammonium acid salts of acetic acids. Hydrogen bonding in solids and solutions, J. Mol. Struct. 140 (1986) 269-279, doi:10.1016/0022-2860(86) 87010-7.

[26] R.G. Treble, K.E. Johnson, E. Tosh, The volatilities and conductivities of ionic liquids - GC-MS methodology and preliminary studies of acetic acid - base systems, Can. J. Chem. 84 (2006) 915-924, doi:10.1139/V06-098.

[27] A. Banerjee, K. Ibsen, T. Brown, R. Chen, C. Agatemor, S. Mitragotri, Definitions of ionic liquids and deep eutectic solvents, Proc. Natl. Acad. Sci. U. S. A. 115 (2018) E11000-E11001, doi:10.1073/pnas.1815526115.

[28] A. Banerjee, K. Ibsen, T. Brown, R. Chen, C. Agatemor, S. Mitragotri, Ionic liquids for oral insulin delivery, Proc. Natl. Acad. Sci. U. S. A. 115 (2018) 7296-7301, doi:10.1073/pnas.1722338115. 
[29] A. Banerjee, K. Ibsen, Y. Iwao, M. Zakrewsky, S. Mitragotri, Transdermal protein delivery using choline and geranate (CAGE) deep eutectic solvent, Adv. Healthc. Mater. 6 (2017) 1601411, doi:10.1002/adhm.201601411.

[30] M. Zakrewsky, A. Banerjee, S. Apte, T.L. Kern, M.R. Jones, R.E.D. Sesto, A.T. Koppisch, D.T. Fox, S. Mitragotri, Choline and geranate deep eutectic solvent as a broad-spectrum antiseptic agent for preventive and therapeutic applications, Adv. Healthc. Mater. 5 (2016) 1282-1289, doi:10.1002/adhm.201600086.

[31] M. Zakrewsky, K.S. Lovejoy, T.L. Kern, T.E. Miller, V. Le, A. Nagy, A.M. Goumas, R.S. Iyer, R.E. DelSesto, A.T. Koppisch, D.T. Fox, S. Mitragotri, Ionic liquids as a class of materials for transdermal delivery and pathogen neutralization, Proc. Natl. Acad. Sci. U. S. A. 111 (2014) 13313-13318, doi:10.1073/pnas.1403995111.

[32] M.A.R. Martins, S.P. Pinho, J.A.P. Coutinho, Insights into the nature of eutectic and deep eutectic mixtures, J. Solution Chem. 48 (2019) 962-982, doi:10.1007/ s10953-018-0793-1.

[33] H.S. van Klooster, W.A. Douglas, The system acetic acid-triethylamine, J. Phys. Chem. 49 (1945) 67-70, doi:10.1021/j150440a002.

[34] F. Kohler, E. Liebermann, G. Miksch, C. Kainz, On the thermodynamics of the acetic acid-triethylamine system, J. Phys. Chem. 76 (1972) 2764-6768, doi:10. 1021/j100663a025.
[35] Y.Q. Lv, Y. Guo, X.Y. Luo, H.R. Li, Infrared spectroscopic study on chemical and phase equilibrium in triethylammonium acetate, Sci. China Chem. 55 (2012) 1688-1694, doi:10.1007/s11426-012-4634-6.

[36] P. Berton, S.P. Kelley, H. Wang, R.D. Rogers, Elucidating the triethylammonium acetate system: Is it molecular or is it ionic? J. Mol. Liq. 269 (2018) 126-131, doi:10.1016/j.molliq.2018.08.006.

[37] F. Kohler, H. Atrops, H. Kalali, E. Liebermann, E. Wilhelm, F. Ratkovics, T. Salamon, Molecular interactions in mixtures of carboxylic acids with amines. 1. Melting curves and viscosities, J. Phys. Chem. 85 (1981) 2520-2524, doi:10. $1021 / j 150617 a 021$.

[38] K. Orzechowski, J. Gliński, U. Kaatze, Acoustic and dielectric properties of propionic acid + amine mixtures, J. Phys. IV. 129 (2005) 73-78, doi:10.1051/jp4: 2005129016.

[39] K. Orzechowski, M. Pajdowska, M. Czarnecki, U. Kaatze, Complexation and proton transfer in the binary system propionic acid-triethylamine evidence from the composition dependencies of mixture properties, J. Mol. Liq. 133 (2007) 11-16, doi:10.1016/j.molliq.2006.05.007.

[40] G.J. Maximo, R.J.B.N. Santos, J.A. Lopes-da-Siva, M.C. Costa, A.J.A. Meirelles, J.A.P. Coutinho, Lipidic protic ionic liquid crystals, ACS Sustain. Chem. Eng. 2 (2014) 672-682, doi:10.1021/sc400365h. 